\title{
Fractal analysis of the relationship between the visual complexity of laser show pictures and a human psychophysiological state
}

\author{
Nikolai Matveev ${ }^{1}$, Aleksandra Sherstobitova ${ }^{2}$, and Olga Gerasimova ${ }^{1}$ \\ ${ }^{1}$ Department of "The Higher School of Lighting Design", Saint Petersburg National Research University of Information \\ Technologies, Mechanics and Optics (ITMO University), Saint-Petersburg, Russia \\ ${ }^{2}$ Department of Light Technologies and Optoelectronics, Saint Petersburg National Research University of Information \\ Technologies, Mechanics and Optics (ITMO University), Saint-Petersburg, Russia
}

\begin{abstract}
This paper presents the results of the fractal analysis of the relationship between the visual complexity of laser show pictures and its human aesthetic perception. Recommendations on the use of laser effects in the art therapy are presented
\end{abstract}

Keywords: psychophysiology, laser graphics, fractal dimension

\section{Introduction}

Today, many people are exposed to stress and its negative impact. In this respect, there is a need to correct a human psychophysiological state (and not only by medical means). The application of different audiovisual systems (Theta Mind's Eye, 2016; Mind Machines, 2016) can significantly improve people's quality of life. These systems can stimulate physiological and emotional activity of humans. Not only they have a relaxing effect, but also help people reduce anxiety and boost their productivity.

Some systems use flashes of light and rhythmic tones to affect the brain. Modern devices based on this principle consist of LED (light-emitting diode) glasses. The visual content of audiovisual systems includes pictures of nature or abstract images. The latter can be created by hand or generated by program code (Mental Rhythm, 2016). Fractal algorithms are often used to generate abstract graphics.

The term "fractal" was first introduced by B. Mandelbrot. It refers to a specific geometrical structure which has a property of self-similarity. Such objects can often be found in nature (trees, circulatory system, etc.) (Forsythe et al., 2011; Bourke, 2016). Today, fractal theory and fractal methods are used in engineering, medicine, art, and other fields. The fractal dimension $\mathrm{D}$ is the basic parameter for quantitative analysis of fractal forms. The Hausdorff dimension and the box dimension are mostly used in experimental science. The latter is defined by:

$$
D=-\lim _{\varepsilon \rightarrow 0} \frac{\log N(\varepsilon)}{\log \varepsilon}
$$

where $N(\varepsilon)$ is the minimum number of "balls" of radius $\varepsilon$ required to cover a certain set (Crownover, 1995).

Today, the fractal dimension is a qualitative aesthetic measure of the visual complexity of paintings and artworks (Forsythe et al., 2011; Mureika, 2005; Taylor et al., 2007; Abbott, 2017). R. P. Taylor, A. Micolich, D. Jonas and other researchers discovered fractal structures in abstract paintings and found the relationship between the fractal dimension of artworks and their human visual perception (Taylor et al., 2001). Thus, paintings with the 1.3-1.5 fractal dimension were aesthetically more preferable to observers than the ones in the fractal dimension of 1.1-1.2 and 1.6-1.9. R. Taylor has also demonstrated beneficial effects of fractal forms on a human psychophysiological state (Forsythe et al., 2011; Taylor et al., 2001).

Laser projection systems gain popularity due to specific characteristics of laser radiation (monochromaticity, coherence, brightness, etc.). They are widely used in the entertainment industry and audiovisual art. These systems enable technicians to create various visual effects. The positive effects of laser projection relaxation methods were previously described by N. Matveev (Matveev, 2016). This paper presents a fractal analysis of the visual complexity of laser show pictures and their impact on human emotional state. 
Fractal methods have been used for the quantitative and qualitative analysis of paintings for a long time (Forsythe et al., 2011; Mureika, 2005; Taylor et al., 2007; Abbott, 2017; Taylor et al., 2001). In this paper, we present the results of the original study on the use of laser show pictures.

\section{Method}

Several types of laser effects are used in light shows: lumia, beam and screen effects. For lumia effects, the laser beam passing through heterogeneous media creates different abstract shapes (interference patterns). To produce beam effects, technicians use smoke or dust and control the laser beam with mirrors, diffraction gratings, etc. For screen effects, laser graphics, texts and animated images are drawn by the laser beam on the projection surface (Daukantas, 2010).

We made pictures of different laser effects used in "Lux Aeterna Theatre" (Russia, Saint-Petersburg) (Lux Aeterna Theatre, 2017). These effects were created by laser technicians using Pangolin software (Pangolin Laser Systems, 2017). We selected 45 images that are most relevant for the purposes of our experiment and divided them into 3 sets of 15 images, where each set represented the corresponding laser effect. Laser show pictures are shown in Figure 1.
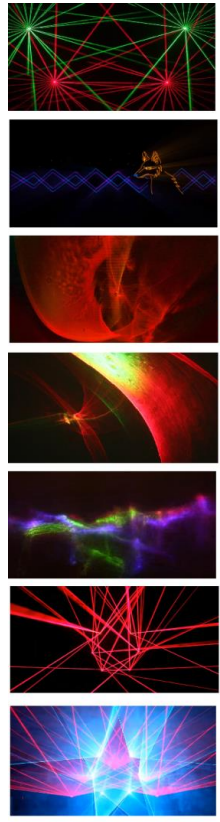
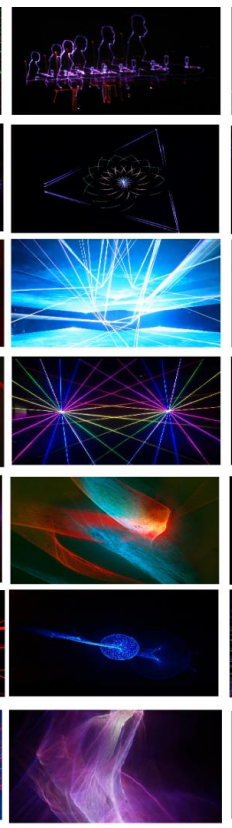
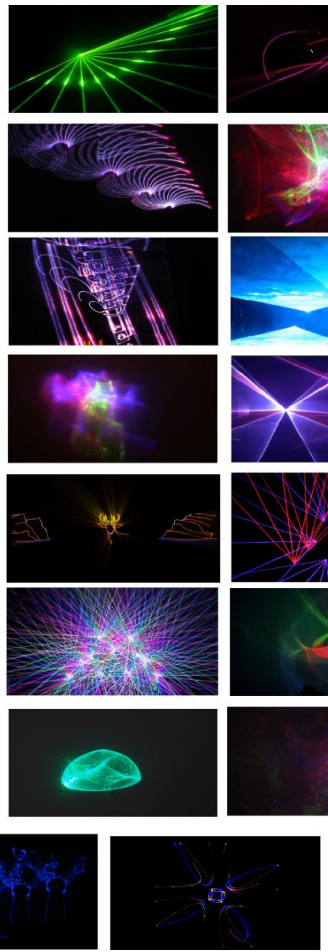
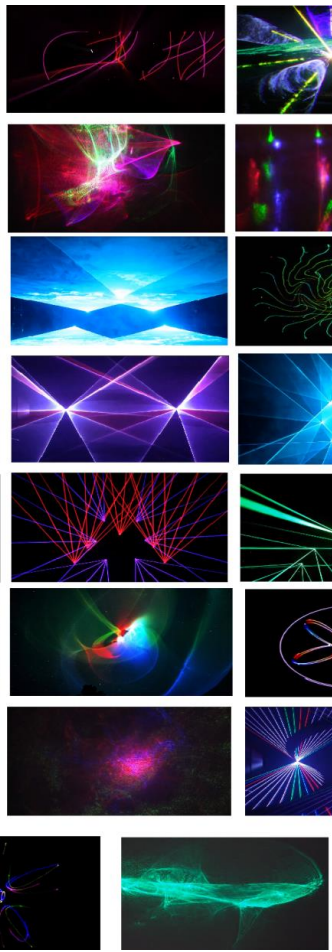
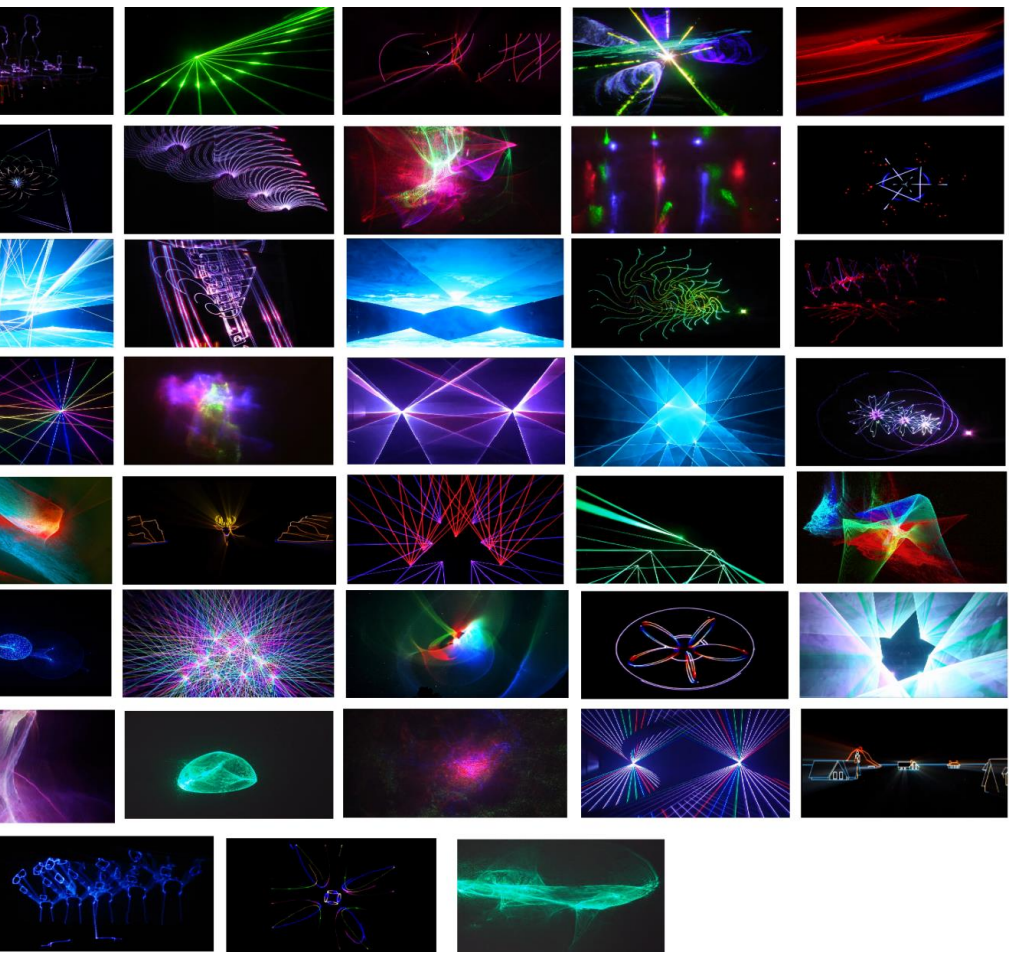

Fig. 1. Laser show images

One hundred and sixty-one participants (men and women aged 17-48 years) in took part in the study. Of these, 44 participants were from the art field and 117 participants did not have any artistic background. Laser show images were presented to the respondents in random order. The participants were asked to rate their emotional and relaxation experience and the attractiveness of the laser show images on a scale from -2 to +2 . Next, we applied the fractal theory in order to establish a relationship between the visual complexity of laser show pictures and their human visual perception. The "box-counting" dimension $D$ was calculated for our images using an imageprocessing software ImageJ (ImageJ, 2016). The color pictures were converted to black and white (monochrome) images using the Canny edge detection algorithm (Canny, 1986). The obtained images were placed on a grid scale, in which all boxes had a fixed size $\varepsilon$. The number of boxes $N(\varepsilon)$ that the image crossed was counted by using the ImageJ software. Next, we reduced the size of boxes and repeated the procedure several times. The fractal dimension $D$ was determined from the slope of the graph, where the $x$-axis was the $\log (\varepsilon)$ and the $y$-axis was $\log (N(\varepsilon))$. 
Thus:

$$
D=-\operatorname{tg} \alpha
$$

where $\alpha$ is the angle of the slope.

We calculated $D$ values of all pictures for different types of laser effects (Table 1).

Table 1. $D$ values of laser show pictures for different types of laser effects

\begin{tabular}{|c|c|c|c|}
\hline Type of laser effect & $D_{\min }$ & $D_{\max }$ & $D_{\text {mean }}$ \\
\hline Lumia effects & 1.296 & 1.657 & 1.490 \\
\hline Beam effects & 1.198 & 1.621 & 1.333 \\
\hline Screen effects & 1.065 & 1.587 & 1.331 \\
\hline
\end{tabular}

Next, we calculated the Spearman correlation coefficients (Wayne, 1989) between $D$ values of the laser show pictures and the respondents' reaction to them (Table 2).

Table 2. The correlation between $D$ values of the laser show pictures and the respondents' reaction to different types of laser effects

\begin{tabular}{|c|c|c|c|}
\hline \multirow{2}{*}{ Type of laser effect } & \multicolumn{2}{|c|}{ Spearman correlation coefficient } \\
\cline { 2 - 4 } & Attractiveness & Emotional effect & Relaxation effect \\
\hline Lumia effects & -0.286 & -0.120 & -0.283 \\
\hline Beam effects & 0.211 & 0.202 & 0.039 \\
\hline Screen effects & 0.110 & 0.119 & 0.039 \\
\hline
\end{tabular}

\section{Results and discussion}

The results of our calculation showed that lumia show pictures had maximum values of fractal dimension $\left(D_{\text {mean }}=\right.$ $1.499)$, whereas beam and screen show pictures had approximately equal $D$ values $\left(D_{\text {mean }}=1.333\right.$ and $D_{\text {mean }}=1.331$, accordingly). Thus, lumia show pictures are more complex than beam and screen ones.

The low correlation between the visual complexity of pictures and the respondents' reaction to them exposes individual preferences for abstract images. The negative correlation coefficients obtained for the lumia effects and the positive relation established for the beam effects are of great importance and point to the following:

1. On the one hand, the structure of the lumia show pictures is complex (the maximum $D$ value falls within the low preference range and the minimum $D$ value is close to the high preference range) so the respondents preferred simpler lumia effects;

2. On the other hand, the minimum $D$ value of the beam show pictures is below the high preference range, so the respondents preferred more complex beam effects.

The obtained results support the theory of a relationship between the fractal dimension and aesthetic judgment (Forsythe et al., 2011; Mureika, 2005; Taylor et al., 2007; Abbott, 2017; Taylor et al., 2001).

In our analysis, we did not take into account the color-grade of the pictures used. In addition, there were some problems with the binarization of the images, as the areas with solid color (generally, with intensity gradients) were interpreted as image "noise". On the one hand, the structure of images proved to more complex (the $D$ value increased); on the other hand, the intensity gradients used for artistic expression affected the complexity of images. Further research is required to explore the impact of the intensity gradients on the visual complexity of the scene. 


\section{Conclusions}

Our research has confirmed the relationship between the visual complexity of laser show pictures and their human psychophysiological perception. However, the individual preferences for abstract images are highly subjective. Therefore, the fractal dimension as such cannot define the aesthetic value of artworks. It should however be noted that the visual complexity of screen effects differs considerably from lumia to beam effects. Screen effects are more suited for the entertainment industry, whereas lumia and beam effects are more relevant for audiovisual art. The latter can be used to create the visual content for relaxation and anxiety management.

\section{References}

1. Abbott A. (2017). Fractal secrets of Rorschach's famed ink blots revealed. Nature. doi:10.1038/nature.2017.21473.

2. Bourke P. (2016). Fractals, Chaos. Retrieved 2016, from http://paulbourke.net/fractals/.

3. Canny J. A. (1986). Computational Approach to Edge Detection. IEEE transactions on pattern analysis and machine intelligence, 8 (5), 679-698.

4. Crownover R. M. (1995). Introduction to Fractals and Chaos. London: Jones and Bartlett Publishers, Inc., $306 \mathrm{p}$.

5. Daukantas P. (2010). A short history of laser light show. Optics and Photonics News, 21 (5), 42-47.

6. Forsythe A., Nadal M., Sheehy N., Cela-Conde C. J., Sawey M. (2011). Predicting beauty: Fractal dimension and visual complexity in art. British Journal of Psychology, 102 (1), 49-70.

7. ImageJ: Image Processing and Analysis in Java. Retrieved 2016, from https://imagej.nih.gov/ij/.

8. Lux Aeterna Theater. Retrieved 2017, from https://www.facebook.com/lux.aeterna.theater/.

9. Matveev N. V., Prokopenko V. T., Sapunova N. P., Fridman D. A. (2016). Research into the influence of light-music performances on psychophysiological states. Light \& Engineering, 24 (2), 22-24.

10. Mental Rhythm. Devices for audiovisual stimulation. Russia. Retrieved 2016, from http://www.mentalritm.ru/pages/katsoftDVD2.html.

11. Mind Machines. USA. Retrieved 2016, from http://www.mindmachines.com/theta-minds-eye/.

12. Mureika J. R. (2005). Fractal dimensions in perceptual color space: A comparison study using Jackson Pollock's art. Chaos, 15 (2). 043702.

13. Taylor R. P., Guzman R., Martin T. P., Hall G. D. R., Micolich A. P., Jonas D., Marlow C. A. (2007). Authenticating Pollock Paintings Using Fractal Geometry. Pattern Recognition Letters, 28 (6), 695-702.

14. Taylor R. P., Newell B. R., Sphehar B. Clifford C. (2001). Fractals: a resonance between art and nature? Intersections of art and science. The Proceedings of Symmetry: Art and Science 1, Fifth Interdisciplinary Symmetry Congress and Exhibition of the International Society for the Interdisciplinary Study of Symmetry, 194-197.

15. Theta Mind's Eye: Light and Sound Machine. Theta Voyager Light and Sound Machines. USA. Retrieved 2016, from http://www.thetavoyager.com/mind's\%20eye.htm/.

16. Pangolin Laser Systems. Retrieved 2017, from http://pangolin.com/.

17. Wayne D. W. (1989). Applied nonparametric statistics. USA: Wadsworth Pub Co., 656 p. 